Ibn Al-Haitham Jour. for Pure \& Appl. Sci. 35(1)2022

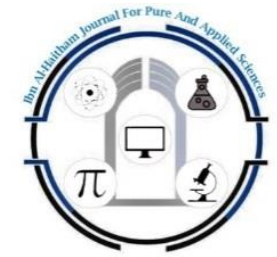

Ibn Al Haitham Journal for Pure and Applied Science

Journal homepage: http://jih.uobaghdad.edu.iq/index.php/j/index

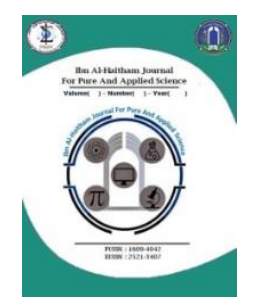

\title{
The Homomorphism of Cubic bipolar ideals of a KU-semigroup
}

\author{
Wisam K. Awad \\ aa.ww21@yahoo.com \\ Department of Mathematics, Ibn- \\ Al-Haitham College of Education, \\ University of Baghdad.
}

\author{
Fatema F. Kareem \\ fa_sa20072000@yahoo.com \\ Department of Mathematics, Ibn-Al- \\ Haitham College of Education, \\ University of Baghdad.
}

Article history: Received 22, June 2021, Accepted 4,October 2021, Published in January 2022.

Doi: $10.30526 / 35.1 .2801$

\begin{abstract}
The idea of a homomorphism of a cubic set of a KU-semigroup is studied and the concept of the product between two cubic sets is defined. And then, a new cubic bipolar fuzzy set in this structure is discussed, and some important results are achieved. Also, the product of cubic subsets is discussed and some theorems are proved.

2010 AMS Classification: 06F35, 03G25, 08A72 .
\end{abstract}

Key words: A KU-semigroup, a cubic sub KU-semigroup, a cubic bipolar $k$-ideal, homomorphism.

\section{Introduction}

In 2009, Prabpayak and Leerawat [1,2] studied a new algebra called a KU-algebra. They introduced homomorphism in a KU-algebra and discussed some recent results. After that, Mostafa et al. [3, 4] studied the concepts of fuzzy KU-ideals and an interval value fuzzy KUideals. In [5] Kareem and Hasan presented the structure of a KU-semigroup and introduced some ideals of this structure. After that, they introduced the fuzzy ideals of this structure in [6]. In [7] Kareem and Talib gave the concept of an interval value fuzzy some ideal in KUsemigroup.

Jun et al. [8,9] introduced the concept of cubic subalgebras/ideals in BCK/BCI-algebras. Yaqoob et al [10] presented a cubic KU-algebra and discussed a few interesting theorems. This work studied the idea of a homomorphism of a cubic set of a KU-semigroup, and a new cubic bipolar fuzzy set in this structure is defined, and some important results are achieved. Also, the product of cubic subsets was discussed, and a few theorems were proved. 


\section{Basic concepts}

We introduce some definitions, propositions and theorems of KU-algebra and KUsemigroup in this part.

Definition2.1[1]. A KU-algebra $(\kappa, *, 0)$ is satisfied the following conditions, for all $\alpha, \beta, \delta \in$ $\aleph$,

$\left(\mathrm{ku}_{1}\right)(\alpha * \beta) *[(\beta * \delta) *(\alpha * \delta)]=0$

$\left(\mathrm{ku}_{2}\right) \alpha * 0=0$

$\left(\mathrm{ku}_{3}\right) 0 * \alpha=\alpha$

$\left(\mathrm{ku}_{4}\right) \alpha * \beta=0$ and $\beta * \alpha$ implies $\alpha=\beta$ and

$\left(\mathrm{ku}_{5}\right) \alpha * \alpha=0$

The relation $\leq$ on a $\mathrm{KU}$-algebra $\mathrm{N}$ is define $\alpha \leq \beta \Leftrightarrow \beta * \alpha=0$.

Example 2.2 [1].The following table define the binary operation $*$ on the set $\aleph=\{0, a, b, c\}$

\begin{tabular}{|c|c|c|c|c|}
\hline$*$ & 0 & $a$ & $b$ & $c$ \\
\hline 0 & 0 & $a$ & $b$ & $c$ \\
\hline$a$ & 0 & 0 & 0 & $b$ \\
\hline$b$ & 0 & $b$ & 0 & $a$ \\
\hline$c$ & 0 & 0 & 0 & 0 \\
\hline
\end{tabular}

Then $(\aleph, *, 0)$ is a KU-algebra.

Theorem2.3[2]. The following axioms are satisfying, in a KU-algebraא.

For all $\alpha, \beta, \delta \in \aleph$,

(1) if $\alpha \leq \beta$ imply $\beta * \delta \leq \alpha * \delta$

(2) $\alpha *(\beta * \delta)=\beta *(\alpha * \delta)$

(3) $((\beta * \alpha) * \alpha) \leq \beta$

Definition2.4[5]. The set $\aleph \neq \varphi$ and two binary operations $*$,o with a constant 0 is named a KU-semigroup if

(I) The triple $(\aleph, *, 0)$ is a $\mathrm{KU}$-algebra

(II) the ordered pair $(\aleph, \circ)$ is a semigroup

(III) $\alpha, \beta, \delta \in \aleph, \alpha \circ(\beta * \delta)=(\alpha \circ \beta) *(\alpha \circ \delta) \operatorname{and}(\alpha * \beta) \circ \delta=(\alpha \circ \delta) *(\beta \circ \delta)$.

Example 2.5[5]. If $\aleph=\{0,1,2,3\}$ is a set and two binary operations $*$ and $\circ$ are defined by the following.

\begin{tabular}{|c|c|c|c|c|}
\hline$*$ & 0 & 1 & 2 & 3 \\
\hline 0 & 0 & 1 & 2 & 3 \\
\hline 1 & 0 & 0 & 0 & 2 \\
\hline 2 & 0 & 2 & 0 & 1 \\
\hline 3 & 0 & 0 & 0 & 0 \\
\hline
\end{tabular}

\begin{tabular}{|c|c|c|c|c|}
\hline$\circ$ & 0 & 1 & 2 & 3 \\
\hline 0 & 0 & 0 & 0 & 0 \\
\hline 1 & 0 & 1 & 0 & 1 \\
\hline 2 & 0 & 0 & 2 & 2 \\
\hline 3 & 0 & 1 & 2 & 3 \\
\hline
\end{tabular}

Then $(\aleph, *, \circ, 0)$ is a KU-semigroup.

Definition 2.6[5]. A non-empty subset $A$ of $\alpha$ is named A subKU-semigroup if it is satisfied $\alpha * \beta, \alpha \circ \beta \in A$, for all $\alpha, \beta \in A$.

Definition2.7[5]. A non-empty subset $I \subseteq \mathcal{W}$ is called an $S$-ideal of $\aleph$, if

(i) $\quad 0 \in I$ 
(ii) $\quad \alpha * \beta \in I$ and $\alpha \in I$ imply $\beta \in I$.

(iii) $\forall \alpha \in \boldsymbol{N}, \boldsymbol{e} \in I$, we have $\alpha \circ \boldsymbol{e} \in I$ and $\boldsymbol{e} \circ \alpha \in I$.

Definition2.8[5]. The set $\varphi \neq A \subseteq \mathcal{K}$ is named a $k$-ideal ofא, if

i) $0 \in I$

ii) $\forall \alpha, \beta, \delta \in \mathrm{N},(\alpha *(\beta * \delta)) \in I, \beta \in \operatorname{Iimply} \alpha * \delta \in I$.

iii) $\forall \alpha \in \boldsymbol{N}, \boldsymbol{e} \in \mathrm{A}$, we have $\alpha \circ \boldsymbol{e} \in \mathrm{A}$ and $\boldsymbol{e} \circ \alpha \in \mathrm{A}$.

Definition2.9[5]. Let $\aleph$ and $\aleph$ 'be two KU-semigroups. A mapping $f: \aleph \rightarrow \aleph$ 'is called a KUsemigroup homomorphism if $f(\alpha * \beta)=f \alpha * f(\beta)$ and $f(\alpha \circ \beta)=f(\alpha) \circ f(\beta)$ for all $\alpha, \beta \in$ $\aleph$. The set $\{\alpha \in \aleph: f(\alpha)=0$ \}is called the kernel of $f$ and denoted byker $f$ Moreover, the set $\left\{f(\alpha) \in \aleph^{\prime}: \alpha \in \aleph\right\}$ is called the image of $f$ and denoted by imf.

We recall that a cubic bipolar valued fuzzy subset in [11] as follows:

Definition 2.10[11]. Let $N$ be a non-empty set. A cubic bipolar set in a set $\aleph$ is the structure $\Omega=\left\{\left\langle\alpha, \tilde{\mu}_{\Omega}^{+}(\alpha), \tilde{\mu}_{\Omega}^{-}(\alpha), \lambda_{\Omega}^{+}(\alpha), \lambda_{\Omega}^{-}(\alpha): \alpha \in \aleph\right\rangle\right\}$ where $N(\alpha)=\left\{\tilde{\mu}_{\Omega}^{+}(\alpha), \tilde{\mu}_{\Omega}^{-}(\alpha)\right\}$ is calledinterval valued bipolar fuzzy set and $K(\alpha)=\left\{\lambda_{\Omega}^{+}(\alpha), \lambda_{\Omega}^{-}(\alpha)\right\}$ is a bipolar fuzzy set. Consider $\tilde{\mu}_{\Omega}^{+}: \aleph \rightarrow D[0,1]$ such that $\tilde{\mu}_{\Omega}^{+}(\alpha)=\left[\delta_{\Omega_{\mathrm{L}}}^{+}(\alpha), \delta_{\Omega_{\mathrm{U}}}^{+}(\alpha)\right]$ and

$\tilde{\mu}_{\Omega}^{-}: \aleph \rightarrow D[-1,0]$ such that $\tilde{\mu}_{\Omega}^{-}(\alpha)=\left[\delta_{\Omega_{\mathrm{L}}}^{-}(\alpha), \delta_{\Omega_{\mathrm{U}}}^{-}(\alpha)\right]$, also $\lambda_{\Omega}^{+}: \aleph \rightarrow[0,1]$, and $\lambda_{\Omega}^{-}: \aleph \rightarrow$ $[-1,0]$ it follows that

$\left.\Omega=\left\{<\alpha,\left\{\left[\delta_{\Omega_{\mathrm{L}}}^{+}(\alpha), \delta_{\Omega_{\mathrm{U}}}^{+}(\alpha)\right],\left[\delta_{\Omega_{\mathrm{L}}}^{-}(\alpha), \delta_{\Omega_{\mathrm{U}}}^{-}(\alpha)\right]\right\}, \lambda_{\Omega}^{+}(\alpha), \lambda_{\Omega}^{-}(\alpha)\right\}>: \alpha \in \aleph\right\}$

It is a cubicbipolar set and can be denoted by $\Omega=\langle N, K\rangle$.

Definition2.11[11]. A Cubic bipolar set $\Omega=\langle N, K\rangle$ is named a cubic bipolar sub KUsemigroup if: $\forall \alpha, \beta \in \aleph$,

(1) $\tilde{\mu}_{\Omega}^{+}(\alpha * \beta) \geq \operatorname{rmin}\left\{\tilde{\mu}_{\Omega}^{+}(\alpha), \tilde{\mu}_{\Omega}^{+}(\beta)\right\}, \tilde{\mu}_{\Omega}^{-}(\alpha * \beta) \leq \operatorname{rmax}\left\{\tilde{\mu}_{\Omega}^{-}(\alpha), \tilde{\mu}_{\Omega}^{-}(\beta)\right\}$

$\lambda_{\Omega}^{+}(\alpha * \beta) \geq \min \left\{\lambda_{\Omega}^{+}(\alpha), \lambda_{\Omega}^{+}(\beta)\right\}, \lambda_{\Omega}^{-}(\alpha * \beta) \leq \max \left\{\lambda_{\Omega}^{-}(\alpha), \lambda_{\Omega}^{-}(\beta)\right\}$,

(2) $\tilde{\mu}_{\Omega}^{+}(\alpha \circ \beta) \geq \operatorname{rmin}\left\{\tilde{\mu}_{\Omega}^{+}(\alpha), \tilde{\mu}_{\Omega}^{+}(\beta)\right\}, \tilde{\mu}_{\Omega}^{-}(\alpha \circ \beta) \leq \operatorname{rmax}\left\{\tilde{\mu}_{\Omega}^{-}(\alpha), \tilde{\mu}_{\Omega}^{-}(\beta)\right\}$

$\lambda_{\Omega}^{+}(\alpha \circ \beta) \geq \min \left\{\lambda_{\Omega}^{+}(\alpha), \lambda_{\Omega}^{+}(\beta)\right\}, \lambda_{\Omega}^{-}(\alpha \circ \beta) \leq \max \left\{\lambda_{\Omega}^{-}(\alpha), \lambda_{\Omega}^{-}(\beta)\right\}$,

Example2.12[11]. If $\boldsymbol{N}=\{0,1,2,3\}$ is a set and two binary operations $*$ and $\circ$ are define by the following.

\begin{tabular}{|c|c|c|c|c|}
\hline$*$ & 0 & 1 & 2 & 3 \\
\hline 0 & 0 & 1 & 2 & 3 \\
\hline 1 & 0 & 0 & 0 & 2 \\
\hline 2 & 0 & 2 & 0 & 1 \\
\hline 3 & 0 & 0 & 0 & 0 \\
\hline
\end{tabular}

\begin{tabular}{|c|c|c|c|c|}
\hline$\circ$ & 0 & 1 & 2 & 3 \\
\hline 0 & 0 & 0 & 0 & 0 \\
\hline 1 & 0 & 1 & 0 & 1 \\
\hline 2 & 0 & 0 & 2 & 2 \\
\hline 3 & 0 & 1 & 2 & 3 \\
\hline
\end{tabular}

$\operatorname{Then}(\aleph, *, \circ, 0)$ is a KU-semigroup. Define $\Omega=\langle N, K\rangle$ as follows

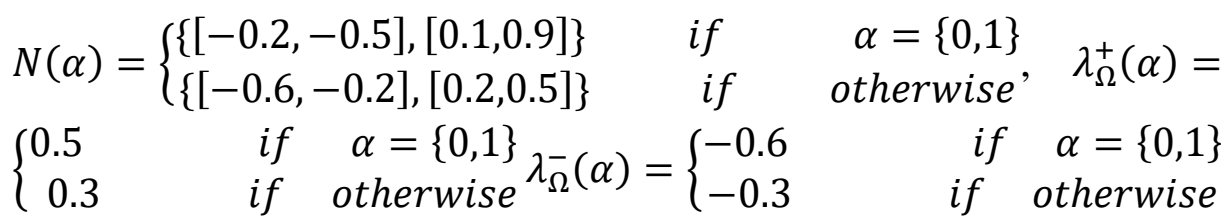
then $\Omega=\langle N, K\rangle$ is a cubic bipolar sub KU-semigroup of $\aleph$.

Definition 2.13[11]. A cubic bipolar set $\Omega=\langle N, K\rangle$ in $\aleph$ is called a cubic bipolar ideal of $\aleph$ if, $\forall \alpha, \beta \in \mathrm{N}$

$\left(\mathbf{B C}_{1}\right) \tilde{\mu}_{\Omega}^{+}(0) \geq \tilde{\mu}_{\Omega}^{+}(\alpha), \lambda_{\Omega}^{+}(0) \geq \lambda_{\Omega}^{+}(\alpha)$ and $\tilde{\mu}_{\Omega}^{-}(0) \leq \tilde{\mu}_{\Omega}^{-}(\alpha), \lambda_{\Omega}^{-}(0) \leq \lambda_{\Omega}^{-}(\alpha)$ 
$(\mathbf{B C}) \tilde{\mu}_{\Omega}^{+}(\beta) \geq \operatorname{rmin}\left\{\tilde{\mu}_{\Omega}^{+}(\alpha * \beta), \tilde{\mu}_{\Omega}^{+}(\alpha)\right\}, \tilde{\mu}_{\Omega}^{-}(\beta) \leq \operatorname{rmax}\left\{\tilde{\mu}_{\Omega}^{-}(\alpha * \beta), \tilde{\mu}_{\Omega}^{-}(\alpha)\right\}$ and $\lambda_{\Omega}^{+}(\beta) \geq \min \left\{\lambda_{\Omega}^{+}(\alpha * \beta), \lambda_{\Omega}^{+}(\alpha)\right\}, \lambda_{\Omega}^{-}(\beta) \leq \max \left\{\lambda_{\Omega}^{-}(\alpha * \beta), \lambda_{\Omega}^{-}(\alpha)\right\}$,

$\left(\mathbf{B C}_{3}\right) \tilde{\mu}_{\Omega}^{+}(\alpha \circ \beta) \geq \operatorname{rmin}\left\{\tilde{\mu}_{\Omega}^{+}(\alpha), \tilde{\mu}_{\Omega}^{+}(\beta)\right\}, \tilde{\mu}_{\Omega}^{-}(\alpha \circ \beta) \leq \operatorname{rmax}\left\{\tilde{\mu}_{\Omega}^{-}(\alpha), \tilde{\mu}_{\Omega}^{-}(\beta)\right\}$ and $\lambda_{\Omega}^{+}(\alpha \circ \beta) \geq \min \left\{\lambda_{\Omega}^{+}(\alpha), \lambda_{\Omega}^{+}(\beta)\right\}, \lambda_{\Omega}^{-}(\alpha \circ \beta) \leq \max \left\{\lambda_{\Omega}^{-}(\alpha), \lambda_{\Omega}^{-}(\beta)\right\}$.

Example 2.14[11]. If $\aleph=\{0,1,2\}$ is a set and two binary operations $*$ and $\circ$ are defined by the following.

\begin{tabular}{|c|l|l|l|}
\hline$*$ & 0 & 1 & 2 \\
\hline 0 & 0 & 1 & 2 \\
\hline 1 & 0 & 0 & 1 \\
\hline 2 & 0 & 1 & 0 \\
\hline
\end{tabular}

\begin{tabular}{|c|l|l|l|}
\hline$\circ$ & 0 & 1 & 2 \\
\hline 0 & 0 & 0 & 0 \\
\hline 1 & 0 & 1 & 0 \\
\hline 2 & 0 & 0 & 2 \\
\hline
\end{tabular}

Then $(\aleph, *, \circ, 0)$ is a KU-semigroup. Define $\Omega=\langle N, K\rangle$ as follows

$N(\alpha)=\left\{\begin{array}{l}\{[-0.3,-0.1],[0.1,0.8]\} \\ \{[-0.7,-0.3],[0.4,0.6]\}\end{array} \quad\right.$ if $\begin{array}{r}\alpha=0 \\ \text { otherwise }\end{array} \quad \lambda_{\Omega}^{+}(\alpha)=$

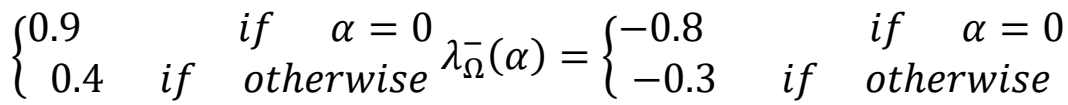

We can easily prove that $\Omega=\langle N, K\rangle$ is a cubic bipolarideal of $\aleph$.

Definition 2.15[11]. A cubicbipolar set $\Omega=\langle N, K\rangle$ in $\aleph$ is called a cubic bipolark-ideal of $\aleph$ if, $\forall \alpha, \beta, \delta \in \aleph$

(a) $\tilde{\mu}_{\Omega}^{+}(0) \geq \tilde{\mu}_{\Omega}^{+}(\alpha), \lambda_{\Omega}^{+}(0) \geq \lambda_{\Omega}^{+}(\alpha)$ and $\tilde{\mu}_{\Omega}^{-}(0) \leq \tilde{\mu}_{\Omega}^{-}(\alpha), \lambda_{\Omega}^{-}(0) \leq \lambda_{\Omega}^{-}(\alpha)$.

(b) $\tilde{\mu}_{\Omega}^{+}(\alpha * \delta) \geq \operatorname{rmin}\left\{\tilde{\mu}_{\Omega}^{+}\left((\alpha *(\beta * \delta)), \tilde{\mu}_{\Omega}^{+}(\beta)\right\}\right.$,

$\tilde{\mu}_{\Omega}^{-}(\alpha * \delta) \leq \operatorname{rmax}\left\{\tilde{\mu}_{\Omega}^{-}\left((\alpha *(\beta * \delta)), \tilde{\mu}_{\Omega}^{-}(\beta)\right\}\right.$ and

$\lambda_{\Omega}^{+}(\alpha * \delta) \geq \min \left\{\lambda_{\Omega}^{+}\left((\alpha *(\beta * \delta)), \lambda_{\Omega}^{+}(\beta)\right\}\right.$,

$\lambda_{\Omega}^{-}(\alpha * \delta) \leq \max \left\{\lambda_{\Omega}^{-}\left((\alpha *(\beta * \delta)), \lambda_{\Omega}^{-}(\beta)\right\}\right.$,

(c) $\tilde{\mu}_{\Omega}^{+}(\alpha \circ \beta) \geq \operatorname{rmin}\left\{\tilde{\mu}_{\Omega}^{+}(\alpha), \tilde{\mu}_{\Omega}^{+}(\beta)\right\}, \tilde{\mu}_{\Omega}^{-}(\alpha \circ \beta) \leq \operatorname{rmax}\left\{\tilde{\mu}_{\Omega}^{-}(\alpha), \tilde{\mu}_{\Omega}^{-}(\beta)\right\}$

$\lambda_{\Omega}^{+}(\alpha \circ \beta) \geq \min \left\{\lambda_{\Omega}^{+}(\alpha), \lambda_{\Omega}^{+}(\beta)\right\}, \lambda_{\Omega}^{-}(\alpha \circ \beta) \leq \max \left\{\lambda_{\Omega}^{-}(\alpha), \lambda_{\Omega}^{-}(\beta)\right\}$.

\section{A Cubic Bipolark-ideal underHomomorphism}

We study some definitions of homomorphism; the product of cubic bipolar $k$-ideals and a cubic bipolar ideal. Also some theorems are discussed.

Definition 3.1. For any $\alpha \in \aleph$. We define a new cubicbipolar fuzzy set $\Omega_{f}=\left(\alpha, \tilde{\mu}_{f}^{+}, \tilde{\mu}_{f}^{-}, \lambda_{f}^{-}, \lambda_{f}^{+}\right)$in $\aleph \quad$ by $\quad \tilde{\mu}_{f}^{-}(\alpha)=\tilde{\mu}^{-}(f(\alpha)) \quad$ and $\tilde{\mu}_{f}^{+}(\alpha)=\tilde{\mu}^{+}(f(\alpha)), \lambda_{f}^{-}(\alpha)=$ $\lambda^{-}(f(\alpha))$ and $\lambda_{f}^{+}(\alpha)=\lambda^{+}(f(\alpha))$, where $f: \aleph \rightarrow \aleph^{\prime}$ is a KU-semigroup homomorphism. For short $\Omega_{f}=\left(\alpha, \tilde{\mu}_{f}^{+}, \tilde{\mu}_{f}^{-}, \lambda_{f}^{-}, \lambda_{f}^{+}\right)$is written $\Omega_{f}$ and a cubic bipolar is ACB.

Example3.2. In Example2.14, we have $\boldsymbol{\aleph}^{\prime}=\left\{0^{\prime}, a, b\right\}$ is a set and $f: \aleph \rightarrow \aleph^{\prime}$ is mapping such that $f(\chi)=\chi^{\prime}$ with two tables

\begin{tabular}{|c|l|l|l|}
\hline$*$ & $0^{\prime}$ & $a$ & $b$ \\
\hline $0^{\prime}$ & $0^{\prime}$ & $a$ & $b$ \\
\hline$a$ & $0^{\prime}$ & $0^{\prime}$ & $b$ \\
\hline$b$ & $0^{\prime}$ & $b$ & $0^{\prime}$ \\
\hline
\end{tabular}

\begin{tabular}{|c|l|l|l|}
\hline$\circ$ & $0^{\prime}$ & $a$ & $b$ \\
\hline $0^{\prime}$ & $0^{\prime}$ & $0^{\prime}$ & $0^{\prime}$ \\
\hline$a$ & $0^{\prime}$ & $a$ & $0^{\prime}$ \\
\hline$b$ & $0^{\prime}$ & $0^{\prime}$ & $a$ \\
\hline
\end{tabular}


Then $f: \mathrm{N} \rightarrow \boldsymbol{\aleph}^{\prime}$ is a KU-semigroup homomorphism and $N\left(\chi^{\prime}\right)=$ $\left\{\begin{array}{c}\{[-0.2,-0.1],[0.2,0.9]\} \\ \{[-0.8,-0.2],[0.3,0.5]\}\end{array} \quad\right.$ if $\begin{array}{c}\chi=0 \\ \text { otherwise }\end{array}, \quad \lambda_{\Omega}^{+}(x)=\left\{\begin{array}{ccc}0.6 & \text { if } \chi=0 \\ 0.2 & \text { if } \text { otherwise }\end{array}\right.$ $\lambda_{\Omega}^{-}(x)=\left\{\begin{array}{l}-0.9 \quad \text { if } \quad \chi=0 \\ -0.4 \quad \text { if } \text { otherwise }\end{array}\right.$

We have: $\tilde{\mu}_{f}^{-}(0)=\tilde{\mu}^{-}(f(0))=\tilde{\mu}^{-}\left(0^{\prime}\right)=[-0.2,-0.1]$ and $\tilde{\mu}_{f}^{-}(1)=\tilde{\mu}^{-}(f(1))=\tilde{\mu}^{-}(a)=[-0.8,-0.2]$ and so on.

Theorem 3.3. Let $f: \aleph \rightarrow \aleph^{\prime}$ be a KU-semigroup homomorphism and onto mapping. Then $\Omega_{f}$ is ACB $k$-ideal of $\alpha$ 'if and only if $\Omega_{f}$ is ACB $k$-ideal of $\aleph$.

Proof. For any $\alpha^{\prime} \in \mathcal{N}^{\prime}$ there exists $\alpha \in \mathbb{N}$ such that $f(\alpha)=\alpha^{\prime}$, we have

$$
\tilde{\mu}_{f}^{+}(0)=\tilde{\mu}^{+}(f(0))=\tilde{\mu}^{+}\left(0^{\prime}\right) \geq \tilde{\mu}^{+}(\alpha)=\tilde{\mu}^{+}(f(\alpha))=\tilde{\mu}_{f}^{+}(\alpha)
$$

$\operatorname{And} \tilde{\mu}_{f}^{-}(0)=\tilde{\mu}^{-}(f(0))=\tilde{\mu}^{-}\left(0^{\prime}\right) \leq \tilde{\mu}^{-}\left(\alpha^{\prime}\right)=\tilde{\mu}^{-}(f(\alpha))=\tilde{\mu}_{f}^{-}(\alpha)$.

Also, $\lambda_{f}^{+}(0)=\lambda^{+}(f(0))=\lambda^{+}\left(0^{\prime}\right) \geq \lambda^{+}(\alpha)=\lambda^{+}(f(\alpha))=\lambda_{f}^{+}(\alpha)$

And $\lambda_{f}^{-}(0)=\lambda^{-}(f(0))=\lambda^{-}\left(0^{\prime}\right) \leq \lambda^{-}\left(\alpha^{\prime}\right)=\lambda^{-}(f(\alpha))=\lambda_{f}^{-}(\alpha)$.

Let $\alpha, \delta \in \mathrm{N}, \gamma^{\prime} \in \mathrm{N}^{\prime}$ then there exists $\beta \in \mathrm{N}$ such that $f(\beta)=\beta^{\prime}$.

We have

$$
\begin{aligned}
\tilde{\mu}_{f}^{+}(\alpha * \delta)=\tilde{\mu}^{+} & (f(\alpha * \delta))=\tilde{\mu}^{+}(f(\alpha) * f(\delta)) \geq \operatorname{rmin}\left\{\tilde{\mu}^{+}\left(f(\alpha) *\left(\beta^{\prime} * f(\delta)\right), \tilde{\mu}^{+}(\beta)\right\}\right. \\
& =\operatorname{rmin}\left\{\tilde{\mu}^{+}(f(\alpha) *(f(\beta) * f(\delta))\}, \tilde{\mu}^{+}(f(\beta)\}\right. \\
& =\operatorname{rmin}\left\{\tilde{\mu}_{f}^{+}(\alpha *(\beta * \delta)), \tilde{\mu}_{f}^{+}(\beta)\right\} .
\end{aligned}
$$

And

$$
\begin{aligned}
\tilde{\mu}_{f}^{-}(\alpha * \delta)=\tilde{\mu}^{-} & (f(\alpha * \delta))=\tilde{\mu}^{-}(f(\alpha) * f(\delta)) \leq \operatorname{rmax}\left\{\tilde{\mu}^{-}\left(f(\alpha) *\left(\beta^{\prime} * f(\delta)\right), \tilde{\mu}^{-}\left(\beta^{\prime}\right)\right\}\right. \\
& =\operatorname{rmax}\left\{\tilde{\mu}^{-}(f(\alpha) *(f(\beta) * f(\delta))\}, \tilde{\mu}^{-}(f(\beta)\}\right. \\
& =\operatorname{rmax}\left\{\tilde{\mu}_{f}^{-}(\alpha *(\beta * \delta)), \tilde{\mu}_{f}^{-}(\beta)\right\} .
\end{aligned}
$$

Also,

$$
\begin{array}{r}
\lambda_{f}^{+}(\alpha * \delta)=\lambda^{+}(f(\alpha * \delta))=\lambda^{+}(f(\alpha) * f(\delta)) \geq \min \left\{\lambda^{+}\left(f(\alpha) *\left(\beta^{\prime} * f(\delta)\right), \lambda^{+}\left(\beta^{\prime}\right)\right\}\right. \\
=\min \left\{\lambda^{+}(f(\alpha) *(f(\beta) * f(\delta))\}, \lambda^{+}(f(\beta)\}=\min \left\{\lambda_{f}^{+}(\alpha *(\beta * \delta)), \lambda_{f}^{+}(\beta)\right\} .\right.
\end{array}
$$

And

$$
\begin{aligned}
\lambda_{f}^{-}(\alpha * \delta)=\lambda^{-} & (f(\alpha * \delta))=\lambda^{-}(f(\alpha) * f(\delta)) \leq \max \left\{\lambda^{-}\left(f(\alpha) *\left(\beta^{\prime} * f(\delta)\right), \lambda^{-}\left(\beta^{\prime}\right)\right\}\right. \\
& =\max \left\{\lambda^{-}(f(\alpha) *(f(\beta) * f(\delta))\}, \lambda^{-}(f(\beta)\}\right. \\
& =\max \left\{\lambda_{f}^{-}(\alpha *(\beta * \delta)), \lambda_{f}^{-}(\beta)\right\} .
\end{aligned}
$$

And the condition (c) is

$$
\begin{gathered}
\tilde{\mu}_{f}^{+}(\alpha \circ \beta)=\tilde{\mu}^{+}(f(\alpha \circ \beta))=\tilde{\mu}^{+}(f(\alpha) \circ f(\beta)) \geq \operatorname{rmin}\left\{\tilde { \mu } ^ { + } \left(f(\alpha), \tilde{\mu}^{+}(f(\beta)\}\right.\right. \\
=\operatorname{rmin}\left\{\tilde{\mu}_{f}^{+}(\alpha), \tilde{\mu}_{f}^{+}(\beta)\right\}
\end{gathered}
$$

And

$$
\begin{gathered}
\tilde{\mu}_{f}^{-}(\alpha \circ \beta)=\tilde{\mu}^{-}(f(\alpha \circ \beta))=\tilde{\mu}^{-}(f(\alpha) \circ f(\beta)) \leq \operatorname{rmax}\left\{\tilde { \mu } ^ { - } \left(f(\alpha), \tilde{\mu}^{-}(f(\beta)\}\right.\right. \\
=\operatorname{rmax}\left\{\tilde{\mu}_{f}^{-}(\alpha), \tilde{\mu}_{f}^{-}(\beta)\right\}
\end{gathered}
$$

Also,

$$
\begin{gathered}
\lambda_{f}^{+}(\alpha \circ \beta)=\lambda^{+}(f(\alpha \circ \beta))=\lambda^{+}(f(\alpha) \circ f(\beta)) \geq \min \left\{\lambda ^ { + } \left(f(\alpha), \lambda^{+}(f(\beta)\}\right.\right. \\
=\min \left\{\lambda_{f}^{+}(\alpha), \lambda_{f}^{+}(\beta)\right\}
\end{gathered}
$$


And

$$
\begin{gathered}
\lambda_{f}^{-}(\alpha \circ \beta)=\lambda^{-}(f(\alpha \circ \beta))=\lambda^{-}(f(\alpha) \circ f(\beta)) \leq \max \left\{\lambda ^ { - } \left(f(\alpha), \lambda^{-}(f(\beta)\}\right.\right. \\
=\max \left\{\lambda_{f}^{-}(\alpha), \lambda_{f}^{-}(\beta)\right\}
\end{gathered}
$$

Conversely, since $f: \aleph \rightarrow \aleph^{\prime}$ is an onto mapping, then for any $\alpha, \beta, \delta \in \aleph^{\prime}$.

It follows that, there exists $\boldsymbol{a}, \boldsymbol{b}, \boldsymbol{c} \in$ Nsuch that $f(\boldsymbol{a})=\alpha, f(\boldsymbol{b})=\beta$ and $f(\boldsymbol{c})=\delta$. We have

$$
\begin{aligned}
\tilde{\mu}_{f}^{+}(\alpha * \delta)=\tilde{\mu}^{+} & (f(\boldsymbol{a}) * f(\boldsymbol{c})))=\tilde{\mu}^{+}(f(\boldsymbol{a} * \boldsymbol{c}))=\tilde{\mu}_{f}^{+}(\boldsymbol{a} * \boldsymbol{c}) \\
& \geq \operatorname{rmin}\left\{\tilde{\mu}_{f}^{+}(\boldsymbol{a} *(\boldsymbol{b} * \boldsymbol{c})), \tilde{\mu}_{f}^{+}(\boldsymbol{b})\right\} \\
& =\operatorname{rmin}\left\{\tilde{\mu}^{+}(f(\boldsymbol{a}) *(f(\boldsymbol{b}) * f(\boldsymbol{c}))\}, \tilde{\mu}^{+}(f(\boldsymbol{b})\}\right. \\
& =\operatorname{rmin}\left\{\tilde{\mu}^{+}(\alpha *(\beta * \delta)), \tilde{\mu}^{+}(\beta)\right\} .
\end{aligned}
$$

And

$$
\begin{aligned}
\tilde{\mu}_{f}^{-}(\alpha * \delta)=\tilde{\mu}^{-} & (f(\boldsymbol{a}) * f(\boldsymbol{c})))=\tilde{\mu}^{-}(f(\boldsymbol{a} * \boldsymbol{c}))=\tilde{\mu}_{f}^{-}(\boldsymbol{a} * \boldsymbol{c}) \\
& \leq \operatorname{rmax}\left\{\tilde{\mu}_{f}^{-}(\boldsymbol{a} *(\boldsymbol{b} * \boldsymbol{c})), \tilde{\mu}_{f}^{-}(\boldsymbol{b})\right\} \\
& =\operatorname{rmax}\left\{\tilde{\mu}^{-}(f(\boldsymbol{a}) *(f(\boldsymbol{b}) * f(\boldsymbol{c}))\}, \tilde{\mu}^{-}(f(\boldsymbol{b})\}\right. \\
& =\operatorname{rmax}\left\{\tilde{\mu}^{-}(\alpha *(\beta * \delta)), \tilde{\mu}^{-}(\beta)\right\} .
\end{aligned}
$$

Also,

$$
\begin{array}{r}
\left.\lambda_{f}^{+}(\alpha * \delta)=\lambda^{+}(f(\boldsymbol{a}) * f(\boldsymbol{c}))\right)=\lambda^{+}(f(\boldsymbol{a} * \boldsymbol{c}))=\lambda_{f}^{+}(\boldsymbol{a} * \boldsymbol{c}) \geq \min \left\{\lambda_{f}^{+}(\boldsymbol{a} *(\boldsymbol{b} * \boldsymbol{c})), \lambda_{f}^{+}(\boldsymbol{b})\right\} \\
=\min \left\{\lambda^{+}(f(\boldsymbol{a}) *(f(\boldsymbol{b}) * f(\boldsymbol{c}))\}, \lambda^{+}(f(\boldsymbol{b})\}=\min \left\{\lambda^{+}(\alpha *(\beta * \delta)), \lambda^{+}(\beta)\right\} .\right.
\end{array}
$$

And

$$
\begin{aligned}
\lambda_{f}^{-}(\alpha * \delta)=\lambda^{-} & (f(\boldsymbol{a}) * f(\boldsymbol{c})))=\lambda^{-}(f(\boldsymbol{a} * \boldsymbol{c}))=\lambda_{f}^{-}(\boldsymbol{a} * \boldsymbol{c}) \\
& \leq \max \left\{\lambda_{f}^{-}(\boldsymbol{a} *(\boldsymbol{b} * \boldsymbol{c})), \lambda_{f}^{-}(\boldsymbol{b})\right\} \\
& =\max \left\{\lambda^{-}(f(\boldsymbol{a}) *(f(\boldsymbol{b}) * f(\boldsymbol{c}))\}, \lambda^{-}(f(\boldsymbol{b})\}\right. \\
& =\max \left\{\lambda^{-}(\alpha *(\beta * \delta)), \lambda^{-}(\beta)\right\} .
\end{aligned}
$$

And the condition (c) is

$$
\left.\tilde{\mu}_{f}^{+}(\alpha \circ \beta)=\tilde{\mu}^{+}(f(\boldsymbol{a}) \circ f(\boldsymbol{b}))\right) \geq \operatorname{rmin}\left\{\tilde{\mu}^{+}(f(\boldsymbol{a})), \tilde{\mu}^{+}(f(\boldsymbol{b})\}=\operatorname{rmin}\left\{\tilde{\mu}_{f}^{+}(\alpha), \tilde{\mu}_{f}^{+}(\beta)\right\}\right.
$$

And

$$
\left.\tilde{\mu}_{f}^{-}(\alpha \circ \beta)=\tilde{\mu}^{-}(f(\boldsymbol{a}) \circ f(\boldsymbol{b}))\right) \leq \operatorname{rmax}\left\{\tilde { \mu } ^ { - } \left(f(\boldsymbol{a}), \tilde{\mu}^{-}(f(\boldsymbol{b})\}=\operatorname{rmax}\left\{\tilde{\mu}_{f}^{-}(\alpha), \tilde{\mu}_{f}^{-}(\beta)\right\}\right.\right.
$$

Also,

$$
\left.\lambda_{f}^{+}(\alpha \circ \beta)=\lambda^{+}(f(\boldsymbol{a}) \circ f(\boldsymbol{b}))\right) \geq \min \left\{\lambda ^ { + } \left(f(\boldsymbol{a}), \lambda^{+}(f(\boldsymbol{b})\}=\min \left\{\lambda_{f}^{+}(\alpha), \lambda_{f}^{+}(\beta)\right\}\right.\right.
$$

And

$$
\left.\lambda_{f}^{-}(\alpha \circ \beta)=\lambda^{-}(f(\boldsymbol{a}) \circ f(\boldsymbol{b}))\right) \leq \max \left\{\lambda ^ { - } \left(f(\boldsymbol{a}), \lambda^{-}(f(\boldsymbol{b})\}=\max \left\{\lambda_{f}^{-}(\alpha), \lambda_{f}^{-}(\beta)\right\}\right.\right.
$$

Therefore $\Omega_{f}$ is ACB $k$-ideal of $\aleph^{\prime}$.

In the following, we introduce the product of the cubic bipolar $k$-ideals and a cubic bipolar ideal as follows.

Definition 3.4. Let $\Omega_{f_{1}}$ and $\Omega_{f_{2}}$ be two CB fuzzy sets of $\aleph$. The product $\Omega_{f_{1}} \times \Omega_{f_{2}}=$ $\left((\alpha, \beta), \quad \tilde{\mu}_{1}^{-} \times \tilde{\mu}_{2}^{-}, \tilde{\mu}_{1}^{+} \times \tilde{\mu}_{2}^{+}, \lambda_{1}^{-} \times \lambda_{2}^{-}, \lambda_{1}^{+} \times \lambda_{2}^{+}\right)$is defined by the following:

$\left(\tilde{\mu}_{1}^{-} \times \tilde{\mu}_{2}^{-}\right)(\alpha, \beta)=\operatorname{rmax}\left\{\tilde{\mu}_{1}^{-}(\alpha), \tilde{\mu}_{2}^{-}(\beta)\right\},\left(\tilde{\mu}_{1}^{+} \times \tilde{\mu}_{2}^{+}\right)(\alpha, \beta)=\operatorname{rmin}\left\{\tilde{\mu}_{1}^{+}(\alpha), \tilde{\mu}_{2}^{+}(\beta)\right\}$ and $\left(\lambda_{1}^{-} \times \lambda_{2}^{-}\right)(\alpha, \beta)=\max \left\{\lambda_{1}^{-}(\alpha), \lambda_{2}^{-}(\beta)\right\},\left(\lambda_{1}^{+} \times \lambda_{2}^{+}\right)(\alpha, \beta)=$ $\min \left\{\lambda_{1}^{+}(\alpha), \lambda_{2}^{+}(\beta)\right\}$ where

$\tilde{\mu}_{1}^{-} \times \tilde{\mu}_{2}^{-}: \aleph \times \aleph \rightarrow[-1,0], \tilde{\mu}_{1}^{+} \times \tilde{\mu}_{2}^{+}: \aleph \times \aleph \rightarrow[0,1]$ and $\lambda_{1}^{-} \times \lambda_{2}^{-}: \aleph \times \aleph \rightarrow[-1,0], \lambda_{1}^{+} \times$ $\lambda_{2}^{+}: \aleph \times \aleph \rightarrow[0,1]$, for all $\alpha, \beta \in \aleph$. 
Theorem 3.5. Let $\Omega_{f_{1}}$ and $\Omega_{f_{2}}$ be two CB $k$-ideals of $\aleph$, then $\Omega_{f_{1}} \times \Omega_{f_{2}}$ is ACB $k$-ideal of $\aleph \times$ $\aleph$.

Proof. Let $(\alpha, \beta) \in \aleph \times \aleph$, we have

$\left(\tilde{\mu}_{1}^{+} \times \tilde{\mu}_{2}^{+}\right)(0,0)=\operatorname{rmin}\left\{\tilde{\mu}_{1}^{+}(0), \tilde{\mu}_{2}^{+}(0)\right\} \geq \operatorname{rmin}\left\{\tilde{\mu}_{1}^{+}(\alpha), \tilde{\mu}_{2}^{+}(\beta)\right\}=\left(\tilde{\mu}_{1}^{+} \times \tilde{\mu}_{2}^{+}\right)(\alpha, \beta)$ and $\left(\tilde{\mu}_{1}^{-} \times \tilde{\mu}_{2}^{-}\right)(0,0)=\operatorname{rmax}\left\{\tilde{\mu}_{1}^{-}(0), \tilde{\mu}_{2}^{-}(0)\right\} \leq \operatorname{rmax}\left\{\tilde{\mu}_{1}^{-}(\alpha), \tilde{\mu}_{2}^{-}(\beta)\right\}=\left(\tilde{\mu}_{1}^{-} \times \tilde{\mu}_{2}^{-}\right)(\alpha, \beta)$.

Let $\left(\alpha_{1}, \alpha_{2}\right),\left(\beta_{1}, \beta_{2}\right)$ and $\left(\delta_{1}, \delta_{2}\right) \in \mathrm{N} \times \boldsymbol{N}$, then

$$
\begin{gathered}
\left(\tilde{\mu}_{1}^{+} \times \tilde{\mu}_{2}^{+}\right)\left(\alpha_{1} * \delta_{1}, \alpha_{2} * \delta_{2}\right)=\operatorname{rmin}\left\{\tilde{\mu}_{1}^{+}\left(\alpha_{1} * \tau_{1}\right), \tilde{\mu}_{2}^{+}\left(\alpha_{2} * \delta_{2}\right)\right\} \\
\quad \geq \operatorname{rmin}\left\{r \min \left\{\tilde{\mu}_{1}^{+}\left(\alpha_{1} *\left(\beta_{1} * \delta_{1}\right)\right), \tilde{\mu}_{1}^{+}\left(\beta_{1}\right)\right\}, r \min \left\{\tilde { \mu } _ { 2 } ^ { + } \left(\alpha_{2}\right.\right.\right. \\
\left.\left.\left.*\left(\beta_{2} * \delta_{2}\right)\right), \tilde{\mu}_{2}^{+}\left(\beta_{2}\right)\right\}\right\}= \\
\operatorname{rmin}\left\{\operatorname { r m i n } \left\{\tilde{\mu}_{1}^{+}\left(\alpha_{1} *\left(\beta_{1} * \delta_{1}\right)\right), \tilde{\mu}_{2}^{+}\left(\alpha_{2} *\left(\beta_{2} * \delta_{2}\right)\right\}, r m i n\left\{\tilde{\mu}_{1}^{+}\left(\beta_{1}\right), \tilde{\mu}_{2}^{+}\left(\beta_{2}\right)\right\}\right.\right. \\
=\operatorname{rmin}\left\{\operatorname{rmin}\left(\tilde{\mu}_{1}^{+} \times \tilde{\mu}_{2}^{+}\right)\left\{\left(\alpha_{1} *\left(\beta_{1} * \delta_{1}\right)\right),\left(\alpha_{2} *\left(\beta_{2} * \delta_{2}\right)\right)\right\},\left\{\left(\tilde{\mu}_{1}^{+} \times \tilde{\mu}_{2}^{+}\right)\left(\beta_{1}, \beta_{2}\right)\right\}\right\}
\end{gathered}
$$

And

$$
\begin{gathered}
\left(\tilde{\mu}_{1}^{-} \times \tilde{\mu}_{2}^{-}\right)\left(\alpha_{1} * \delta_{1}, \alpha_{2} * \delta_{2}\right)=\operatorname{rmax}\left\{\tilde{\mu}_{1}^{-}\left(\alpha_{1} * \delta_{1}\right), \tilde{\mu}_{2}^{-}\left(\alpha_{2} * \delta_{2}\right)\right\} \\
\quad \leq \operatorname{rmax}\left\{\operatorname{rmax}\left\{\tilde{\mu}_{1}^{-}\left(\alpha_{1} *\left(\beta_{1} * \delta_{1}\right)\right), \tilde{\mu}_{1}^{-}\left(\beta_{1}\right)\right\}, \operatorname{rmax}\left\{\tilde { \mu } _ { 2 } ^ { - } \left(\alpha_{2}\right.\right.\right. \\
\left.*\left(\beta_{2} * \delta_{2}\right), \tilde{\mu}_{2}^{-}\left(\beta_{2}\right)\right\}= \\
\operatorname{rmax}\left\{\operatorname { r m a x } \left\{\tilde{\mu}_{1}^{-}\left(\alpha_{1} *\left(\beta_{1} * \delta_{1}\right)\right), \tilde{\mu}_{2}^{-}\left(\alpha_{2} *\left(\beta_{2} * \delta_{2}\right)\right\}, \operatorname{rmax}\left\{\tilde{\mu}_{1}^{-}\left(\beta_{1}\right), \tilde{\mu}_{2}^{-}\left(\beta_{2}\right)\right\}\right.\right. \\
=\operatorname{rmax}\left\{\left(\tilde{\mu}_{1}^{-} \times \tilde{\mu}_{2}^{-}\right)\left\{\left(\alpha_{1} *\left(\beta_{1} * \delta_{1}\right)\right),\left(\alpha_{2} *\left(\beta_{2} * \delta_{2}\right)\right)\right\},\left\{\left(\tilde{\mu}_{1}^{-} \times \tilde{\mu}_{2}^{-}\right)\left(\beta_{1}, \beta_{2}\right)\right\}\right\} .
\end{gathered}
$$

Also,

$$
\begin{aligned}
& \left(\lambda_{1}^{+} \times \lambda_{2}^{+}\right)\left(\alpha_{1} * \delta_{1}, \alpha_{2} * \delta_{2}\right)=\min \left\{\lambda_{1}^{+}\left(\alpha_{1} * \delta_{1}\right), \lambda_{2}^{+}\left(\alpha_{2} * \delta_{2}\right)\right\} \\
& \geq \min \left\{\min \left\{\lambda_{1}^{+}\left(\alpha_{1} *\left(\beta_{1} * \delta_{1}\right)\right), \lambda_{1}^{+}\left(\beta_{1}\right)\right\}, \min \left\{\lambda_{2}^{+}\left(\alpha_{2} *\left(\beta_{2} * \delta_{2}\right)\right), \lambda_{2}^{+}\left(\beta_{2}\right)\right\}\right\} \\
& = \\
& \min \left\{\operatorname { m i n } \left\{\lambda_{1}^{+}\left(\alpha_{1} *\left(\beta_{1} * \delta_{1}\right)\right), \lambda_{2}^{+}\left(\alpha_{2} *\left(\beta_{2} * \delta_{2}\right)\right\}, \min \left\{\lambda_{1}^{+}\left(\beta_{1}\right), \lambda_{2}^{+}\left(\beta_{2}\right)\right\}\right.\right. \\
& =\min \left\{\min \left(\lambda_{1}^{+} \times \lambda_{2}^{+}\right)\left\{\left(\alpha_{1} *\left(\beta_{1} * \delta_{1}\right)\right),\left(\alpha_{2} *\left(\beta_{2} * \delta_{2}\right)\right)\right\},\left\{\left(\lambda_{1}^{+} \times \lambda_{2}^{+}\right)\left(\beta_{1}, \beta_{2}\right)\right\}\right\}
\end{aligned}
$$

And

$$
\begin{aligned}
& \begin{array}{l}
\left(\lambda_{1}^{-} \times \lambda_{2}^{-}\right)\left(\alpha_{1} * \delta_{1}, \alpha_{2} * \delta_{2}\right)=\max \left\{\lambda_{1}^{-}\left(\alpha_{1} * \delta_{1}\right), \lambda_{2}^{-}\left(\alpha_{2} * \delta_{2}\right)\right\} \\
\quad \leq \max \left\{\max \left\{\lambda_{1}^{-}\left(\alpha_{1} *\left(\beta_{1} * \delta_{1}\right)\right), \lambda_{1}^{-}\left(\beta_{1}\right)\right\}, \max \left\{\lambda_{2}^{-}\left(\alpha_{2} *\left(\beta_{2} * \delta_{2}\right), \lambda_{2}^{-}\left(\beta_{2}\right)\right\}\right.\right. \\
\quad= \\
\quad \max \left\{\operatorname { m a x } \left\{\lambda_{1}^{-}\left(\alpha_{1} *\left(\beta_{1} * \delta_{1}\right)\right), \lambda_{2}^{-}\left(\alpha_{2} *\left(\beta_{2} * \delta_{2}\right)\right\}, \max \left\{\lambda_{1}^{-}\left(\beta_{1}\right), \lambda_{2}^{-}\left(\beta_{2}\right)\right\}\right.\right.
\end{array} \\
& =\max \left\{\left(\lambda_{1}^{-} \times \lambda_{2}^{-}\right)\left\{\left(\alpha_{1} *\left(\beta_{1} * \delta_{1}\right)\right),\left(\alpha_{2} *\left(\beta_{2} * \delta_{2}\right)\right)\right\},\left\{\left(\lambda_{1}^{-} \times \lambda_{2}^{-}\right)\left(\beta_{1}, \beta_{2}\right)\right\}\right\} . \\
& \text { And } \quad\left(\tilde{\mu}_{1}^{+} \times \tilde{\mu}_{2}^{+}\right)\left(\alpha_{1} \circ \beta_{1}, \alpha_{2} \circ \beta_{2}\right)=\operatorname{rmin}\left\{\tilde { \mu } _ { 1 } ^ { + } \left(\alpha_{1} \circ\right.\right.
\end{aligned}
$$

$$
\begin{aligned}
& \left(\tilde{\mu}_{1}^{-} \times \tilde{\mu}_{2}^{-}\right)\left(\alpha_{1} \circ \beta_{1}, \alpha_{2} \circ \beta_{2}\right)=\operatorname{rmax}\left\{\tilde{\mu}_{1}^{-}\left(\alpha_{1} \circ \beta_{1}\right), \tilde{\mu}_{2}^{-}\left(\alpha_{2} \circ \beta_{2}\right)\right\} \\
& \leq \operatorname{rmax}\left\{\operatorname{rmax}\left\{\tilde{\mu}_{1}^{-}\left(\alpha_{1}\right), \tilde{\mu}_{1}^{-}\left(\beta_{1}\right)\right\}, \operatorname{rmax}\left\{\tilde{\mu}_{2}^{-}\left(\alpha_{2}\right), \tilde{\mu}_{2}^{-}\left(\beta_{2}\right)\right\}=\right.
\end{aligned}
$$$$
\operatorname{rmax}\left\{\operatorname{rmax}\left\{\tilde{\mu}_{1}^{-}\left(\alpha_{1}\right), \tilde{\mu}_{2}^{-}\left(\alpha_{2}\right)\right\}, \operatorname{rmax}\left\{\tilde{\mu}_{1}^{-}\left(\beta_{1}\right), \tilde{\mu}_{2}^{-}\left(\beta_{2}\right)\right\}\right.
$$

$=\operatorname{rmax}\left\{\left\{\left(\tilde{\mu}_{1}^{-} \times \tilde{\mu}_{2}^{-}\right)\left(\alpha_{1}, \alpha_{2}\right)\right\},\left\{\left(\tilde{\mu}_{1}^{-} \times \tilde{\mu}_{2}^{-}\right)\left(\beta_{1}, \beta_{2}\right)\right\}\right\}$.

Also,

$$
\begin{aligned}
& \left(\lambda_{1}^{+} \times \lambda_{2}^{+}\right)\left(\alpha_{1} \circ \beta_{1}, \alpha_{2} \circ \beta_{2}\right)=\min \left\{\lambda_{1}^{+}\left(\alpha_{1} \circ \beta_{1}\right), \lambda_{2}^{+}\left(\alpha_{2} \circ \beta_{2}\right)\right\} \\
& \geq \min \left\{\min \left\{\lambda_{1}^{+}\left(\alpha_{1}\right), \lambda_{1}^{+}\left(\beta_{1}\right)\right\}, \min \left\{\lambda_{2}^{+}\left(\alpha_{2}\right), \lambda_{2}^{+}\left(\beta_{2}\right)\right\}=\right.
\end{aligned}
$$


Ibn Al-Haitham Jour. for Pure \& Appl. Sci. 35(1)2022

And

$\min \left\{\min \left\{\lambda_{1}^{+}\left(\alpha_{1}\right), \lambda_{2}^{+}\left(\alpha_{2}\right)\right\}, \min \left\{\lambda_{1}^{+}\left(\beta_{1}\right), \lambda_{2}^{+}\left(\beta_{2}\right)\right\}\right.$

$=\min \left\{\left\{\left(\lambda_{1}^{+} \times \lambda_{2}^{+}\right)\left(\alpha_{1}, \alpha_{2}\right)\right\},\left\{\left(\lambda_{1}^{+} \times \lambda_{2}^{+}\right)\left(\beta_{1}, \beta_{2}\right)\right\}\right\}$

$$
\begin{aligned}
& \qquad \begin{array}{l}
\left(\lambda_{1}^{-} \times \lambda_{2}^{-}\right)\left(\alpha_{1} \circ \beta_{1}, \alpha_{2} \circ \beta_{2}\right)=\max \left\{\lambda_{1}^{-}\left(\alpha_{1} \circ \beta_{1}\right), \lambda_{2}^{-}\left(\alpha_{2} \circ \beta_{2}\right)\right\} \\
\leq \max \left\{\max \left\{\lambda_{1}^{-}\left(\alpha_{1}\right), \lambda_{1}^{-}\left(\beta_{1}\right)\right\}, \max \left\{\lambda_{2}^{-}\left(\alpha_{2}\right), \lambda_{2}^{-}\left(\beta_{2}\right)\right\}=\right. \\
\max \left\{\max \left\{\lambda_{1}^{-}\left(\alpha_{1}\right), \lambda_{2}^{-}\left(\alpha_{2}\right)\right\}, \max \left\{\lambda_{1}^{-}\left(\beta_{1}\right), \lambda_{2}^{-}\left(\beta_{2}\right)\right\}\right.
\end{array} \\
& =\max \left\{\left\{\left(\lambda_{1}^{-} \times \lambda_{2}^{-}\right)\left(\alpha_{1}, \alpha_{2}\right)\right\},\left\{\left(\lambda_{1}^{-} \times \lambda_{2}^{-}\right)\left(\beta_{1}, \beta_{2}\right)\right\}\right\} . \\
& \text { Then } \Omega_{f_{1}} \times \Omega_{f_{2}} \text { is ACB } k \text {-ideal of } \aleph \times \aleph .
\end{aligned}
$$

Theorem 3.6.Let $\Omega_{f_{1}}$ and $\Omega_{f_{2}}$ be two CB ideal of KU-semigroupN, such that $\Omega_{f_{1}} \times \Omega_{f_{2}}$ is ACB ideal of $\aleph \times \aleph$. We have

(i) Either $\tilde{\mu}_{1}^{+}(0) \geq \tilde{\mu}_{1}^{+}(\alpha), \tilde{\mu}_{1}^{-}(0) \leq \tilde{\mu}_{1}^{-}(\alpha) \operatorname{or} \tilde{\mu}_{2}^{+}(0) \geq \tilde{\mu}_{2}^{+}(\beta), \tilde{\mu}_{2}^{-}(0) \leq \tilde{\mu}_{2}^{-}(\beta)$, also, $\lambda_{1}^{+}(0) \geq$ $\lambda_{1}^{+}(\alpha), \lambda_{1}^{-}(0) \leq \lambda_{1}^{-}(\alpha)$ or $\lambda_{2}^{+}(0) \geq \lambda_{2}^{+}(\beta), \lambda_{2}^{-}(0) \leq \lambda_{2}^{-}(\beta)$ for all $\alpha, \beta \in \aleph$.

(ii) If $\tilde{\mu}_{1}^{+}(0) \geq \tilde{\mu}_{1}^{+}(\alpha), \tilde{\mu}_{1}^{-}(0) \leq \tilde{\mu}_{1}^{-}(\alpha)$ and $\lambda_{1}^{+}(0) \geq \lambda_{1}^{+}(\alpha), \lambda_{1}^{-}(0) \leq \lambda_{1}^{-}(\alpha)$ for all $\alpha \in \aleph$.

Then either $\tilde{\mu}_{2}^{+}(0) \geq \tilde{\mu}_{1}^{+}(\alpha), \tilde{\mu}_{2}^{-}(0) \leq \tilde{\mu}_{1}^{-}(\alpha)$ and $\lambda_{2}^{+}(0) \geq \lambda_{1}^{+}(\alpha), \lambda_{2}^{-}(0) \leq \lambda_{1}^{-}(\alpha)$ or $\tilde{\mu}_{2}^{+}(0) \geq$ $\tilde{\mu}_{2}^{+}(\beta), \tilde{\mu}_{2}^{-}(0) \leq \tilde{\mu}_{2}^{-}(\beta)$ and $\lambda_{2}^{+}(0) \geq \lambda_{2}^{+}(\beta), \lambda_{2}^{-}(0) \leq \lambda_{2}^{-}(\beta)$ for all $\alpha, \beta \in \mathrm{\aleph}$.

(iii) If $\tilde{\mu}_{2}^{+}(0) \geq \tilde{\mu}_{2}^{+}(\alpha), \tilde{\mu}_{2}^{-}(0) \leq \tilde{\mu}_{2}^{-}(\alpha)$, and $\lambda_{2}^{+}(0) \geq \lambda_{2}^{+}(\alpha), \lambda_{2}^{-}(0) \leq \lambda_{2}^{-}(\alpha)$,for all $\alpha \in \aleph$, then either $\tilde{\mu}_{1}^{+}(0) \geq \tilde{\mu}_{1}^{+}(\alpha), \tilde{\mu}_{1}^{-}(0) \leq \tilde{\mu}_{1}^{-}(\alpha)$, and $\lambda_{1}^{+}(0) \geq \lambda_{1}^{+}(\alpha), \lambda_{1}^{-}(0) \leq \lambda_{1}^{-}(\alpha)$ or $\tilde{\mu}_{1}^{+}(0) \geq$ $\tilde{\mu}_{2}^{+}(\alpha), \tilde{\mu}_{1}^{-}(0) \leq \tilde{\mu}_{2}^{-}(\alpha)$ and $\lambda_{1}^{+}(0) \geq \lambda_{2}^{+}(\alpha), \lambda_{1}^{-}(0) \leq \lambda_{2}^{-}(\alpha)$ for all $\alpha \in \aleph$.

Proof. (i)Suppose that $\tilde{\mu}_{1}^{+}(0) \geq \tilde{\mu}_{1}^{+}(\alpha), \tilde{\mu}_{1}^{-}(0) \leq \tilde{\mu}_{1}^{-}(\alpha)$ and $\tilde{\mu}_{2}^{+}(0) \geq \tilde{\mu}_{2}^{+}(y), \tilde{\mu}_{2}^{-}(0) \leq$ $\tilde{\mu}_{2}^{-}(\beta)$, also, $\lambda_{1}^{+}(0) \geq \lambda_{1}^{+}(\alpha), \lambda_{1}^{-}(0) \leq \lambda_{1}^{-}(\alpha)$ and $\lambda_{2}^{+}(0) \geq \lambda_{2}^{+}(\beta), \lambda_{2}^{-}(0) \leq \lambda_{2}^{-}(\beta)$, for some $\alpha, \beta \in \mathrm{K}$.Then

$$
\left(\tilde{\mu}_{1}^{+} \times \tilde{\mu}_{2}^{+}\right)(\alpha, \beta)=\operatorname{rmin}\left\{\tilde{\mu}_{1}^{+}(\alpha), \tilde{\mu}_{2}^{+}(\beta)\right\} \geq \operatorname{rmin}\left\{\tilde{\mu}_{1}^{+}(0), \tilde{\mu}_{2}^{+}(0)\right\}=\left(\tilde{\mu}_{1}^{+} \times \tilde{\mu}_{2}^{+}\right)(0,0)
$$

And

$$
\left(\tilde{\mu}_{1}^{-} \times \tilde{\mu}_{2}^{-}\right)(\alpha, \beta)=\operatorname{rmax}\left\{\tilde{\mu}_{1}^{-}(\alpha), \tilde{\mu}_{2}^{-}(\beta)\right\} \leq \operatorname{rmax}\left\{\tilde{\mu}_{1}^{-}(0), \tilde{\mu}_{2}^{-}(0)\right\}=\left(\tilde{\mu}_{1}^{-} \times \tilde{\mu}_{2}^{-}\right)(0,0)
$$

Also,

$$
\left(\lambda_{1}^{+} \times \lambda_{2}^{+}\right)(\alpha, \beta)=\min \left\{\lambda_{1}^{+}(\alpha), \lambda_{2}^{+}(\beta)\right\} \geq \min \left\{\lambda_{1}^{+}(0), \lambda_{2}^{+}(0)\right\}=\left(\lambda_{1}^{+} \times \lambda_{2}^{+}\right)(0,0)
$$

And

$$
\left(\lambda_{1}^{-} \times \lambda_{2}^{-}\right)(\alpha, \beta)=\max \left\{\lambda_{1}^{-}(\alpha), \lambda_{2}^{-}(\beta)\right\} \leq \max \left\{\lambda_{1}^{-}(0), \lambda_{2}^{-}(0)\right\}=\left(\lambda_{1}^{-} \times \lambda_{2}^{-}\right)(0,0)
$$

, for all $\alpha, \beta \in \mathrm{N}$. This is a contradiction. Therefore, either $\tilde{\mu}_{1}^{+}(0) \geq \tilde{\mu}_{1}^{+}(\alpha), \tilde{\mu}_{1}^{-}(0) \leq \tilde{\mu}_{1}^{-}(\alpha)$ or $\tilde{\mu}_{2}^{+}(0) \geq \tilde{\mu}_{2}^{+}(\beta), \tilde{\mu}_{2}^{-}(0) \leq \tilde{\mu}_{2}^{-}(\beta)$, also, $\lambda_{1}^{+}(0) \geq \lambda_{1}^{+}(\alpha), \lambda_{1}^{-}(0) \leq \lambda_{1}^{-}(\alpha)$ or $\lambda_{2}^{+}(0) \geq$ $\lambda_{2}^{+}(\beta), \lambda_{2}^{-}(0) \leq \lambda_{2}^{-}(\beta)$ for all $\alpha, \beta \in \aleph$.

(ii)Suppose that $\tilde{\mu}_{2}^{+}(0) \leq \tilde{\mu}_{1}^{+}(\alpha), \tilde{\mu}_{2}^{-}(0) \geq \tilde{\mu}_{1}^{-}(\alpha)$ and $\tilde{\mu}_{2}^{+}(0) \leq \tilde{\mu}_{2}^{+}(\beta), \tilde{\mu}_{2}^{-}(0) \geq \tilde{\mu}_{2}^{-}(\beta)$ also, $\lambda_{2}^{+}(0) \leq \lambda_{1}^{+}(\alpha), \lambda_{2}^{-}(0) \geq \lambda_{1}^{-}(\alpha)$ and $\lambda_{2}^{+}(0) \leq \lambda_{2}^{+}(\beta), \lambda_{2}^{-}(0) \geq \lambda_{2}^{-}(\beta)$, for all $\alpha, \beta \in \aleph$.

Then $\left(\tilde{\mu}_{1}^{+} \times \tilde{\mu}_{2}^{+}\right)(0,0)=\operatorname{rmin}\left\{\tilde{\mu}_{1}^{+}(0), \tilde{\mu}_{2}^{+}(0)\right\}=\tilde{\mu}_{2}^{+}(0)$

And

$$
\left(\tilde{\mu}_{1}^{+} \times \tilde{\mu}_{2}^{+}\right)(\alpha, \beta)=\operatorname{rmin}\left\{\tilde{\mu}_{1}^{+}(\alpha), \tilde{\mu}_{2}^{+}(\beta)\right\} \geq\left\{\tilde{\mu}_{2}^{+}(0), \tilde{\mu}_{2}^{+}(0)\right\}=\tilde{\mu}_{2}^{+}(0)=\left(\tilde{\mu}_{1}^{+} \times \tilde{\mu}_{2}^{+}\right)(0,0)
$$
And $\left(\tilde{\mu}_{1}^{-} \times \tilde{\mu}_{2}^{-}\right)(0,0)=\operatorname{rmax}\left\{\tilde{\mu}_{1}^{-}(0), \tilde{\mu}_{2}^{-}(0)\right\}=\tilde{\mu}_{2}^{-}(0)$.

$\left(\tilde{\mu}_{1}^{-} \times \tilde{\mu}_{2}^{-}\right)(\alpha, \beta)=\operatorname{rmax}\left\{\tilde{\mu}_{1}^{-}(\alpha), \tilde{\mu}_{2}^{-}(\beta)\right\} \leq \operatorname{rmax}\left\{\tilde{\mu}_{2}^{-}(0), \tilde{\mu}_{2}^{-}(0)\right\}=\tilde{\mu}_{2}^{-}(0)=$ $\left(\tilde{\mu}_{1}^{-} \times \tilde{\mu}_{2}^{-}\right)(0,0)$ aaa 


\section{Ibn Al-Haitham Jour. for Pure \& Appl. Sci. 35(1)2022}

Also,

And

$$
\left(\lambda_{1}^{+} \times \lambda_{2}^{+}\right)(0,0)=\min \left\{\lambda_{1}^{+}(0), \lambda_{2}^{+}(0)\right\}=\lambda_{2}^{+}(0)
$$

$\left(\lambda_{1}^{+} \times \lambda_{2}^{+}\right)(\alpha, \beta)=\min \left\{\lambda_{1}^{+}(\alpha), \lambda_{2}^{+}(\beta)\right\} \geq\left\{\lambda_{2}^{+}(0), \lambda_{2}^{+}(0)\right\}=\lambda_{2}^{+}(0)=$ $\left(\lambda_{1}^{+} \times \lambda_{2}^{+}\right)(0,0)$.

And $\left(\lambda_{1}^{-} \times \lambda_{2}^{-}\right)(0,0)=\max \left\{\lambda_{1}^{-}(0), \lambda_{2}^{-}(0)\right\}=\lambda_{2}^{-}(0)$.

$\left(\lambda_{1}^{-} \times \lambda_{2}^{-}\right)(\alpha, \beta)=\max \left\{\lambda_{1}^{-}(\alpha), \lambda_{2}^{-}(\beta)\right\} \leq \max \left\{\lambda_{2}^{-}(0), \lambda_{2}^{-}(0)\right\}=\lambda_{2}^{-}(0)$

$$
=\left(\lambda_{1}^{-} \times \lambda_{2}^{-}\right)(0,0)
$$

. This is a contradiction. Therefore, either $\tilde{\mu}_{2}^{+}(0) \geq \tilde{\mu}_{1}^{+}(\chi), \tilde{\mu}_{2}^{-}(0) \leq \tilde{\mu}_{1}^{-}(\alpha)$ and $\lambda_{2}^{+}(0) \geq$ $\lambda_{1}^{+}(\alpha), \lambda_{2}^{-}(0) \leq \lambda_{1}^{-}(x)$ or $\tilde{\mu}_{2}^{+}(0) \geq \tilde{\mu}_{2}^{+}(\beta), \tilde{\mu}_{2}^{-}(0) \leq \tilde{\mu}_{2}^{-}(\beta)$ and $\lambda_{2}^{+}(0) \geq \lambda_{2}^{+}(\beta), \lambda_{2}^{-}(0) \leq$ $\lambda_{2}^{-}(\beta)$.

(iii)The proof is similar to (ii).

The partial converse of Theorem (3.5) is the following.

Theorem3.7. In a KU-semigroup $\aleph$. If $\Omega_{f_{1}} \times \Omega_{f_{2}}$ is ACB ideal of $\aleph \times \aleph$, then $\Omega_{\mathrm{f}_{1}}$ or $\Omega_{\mathrm{f}_{2}}$ is ACB ideal of $\aleph$.

Proof. By use the Theorem (3.5) (i), without loss of generality we suppose that $\tilde{\mu}_{2}^{+}(0) \geq$ $\tilde{\mu}_{2}^{+}(\alpha), \tilde{\mu}_{2}^{-}(0) \leq \tilde{\mu}_{2}^{-}(\alpha)$, and $\lambda_{2}^{+}(0) \geq \lambda_{2}^{+}(\alpha), \lambda_{2}^{-}(0) \leq \lambda_{2}^{-}(\alpha)$,for all $\alpha \in \kappa$. It follows from Theorem (4.6)(iii) that either then either

$\tilde{\mu}_{1}^{+}(0) \geq \tilde{\mu}_{1}^{+}(\alpha), \tilde{\mu}_{1}^{-}(0) \leq \tilde{\mu}_{1}^{-}(\alpha)$, and $\lambda_{1}^{+}(0) \geq \lambda_{1}^{+} \alpha, \lambda_{1}^{-}(0) \leq \lambda_{1}^{-}(\alpha)$ or $\tilde{\mu}_{1}^{+}(0) \geq \tilde{\mu}_{2}^{+}(\alpha)$, $\tilde{\mu}_{1}^{-}(0) \leq \tilde{\mu}_{2}^{-}(\alpha)$ and $\lambda_{1}^{+}(0) \geq \lambda_{2}^{+}(\alpha), \lambda_{1}^{-}(0) \leq \lambda_{2}^{-}(\alpha)$.

, for all $\alpha \in \mathrm{N}$.

Then $\left(\widetilde{\mu}_{1}^{+} \times \widetilde{\mu}_{2}^{+}\right)(0, \alpha)=\operatorname{rmin}\left\{\widetilde{\mu}_{1}^{+}(0), \widetilde{\mu}_{2}^{+}(\alpha)\right\}=\widetilde{\mu}_{2}^{+}(\alpha)$

$\left(\widetilde{\mu}_{1}^{-} \times \widetilde{\mu}_{2}^{-}\right)(0, \alpha)=\operatorname{rmax}\left\{\widetilde{\mu}_{1}^{-}(0), \widetilde{\mu}_{2}^{-}(\alpha)\right\}=\widetilde{\mu}_{2}^{-}(\alpha)$

Also,

$$
\begin{aligned}
& \begin{aligned}
\left(\lambda_{1}^{+} \times \lambda_{2}^{+}\right)(0, \alpha)= & \min \left\{\lambda_{1}^{+}(0), \lambda_{2}^{+}(\alpha)\right\}=\lambda_{2}^{+}(\alpha) \ldots \ldots(3) \\
& \left(\lambda_{1}^{-} \times \lambda_{2}^{-}\right)(0, \alpha)=\max \left\{\lambda_{1}^{-}(0), \lambda_{2}^{-}(\alpha)\right\}=\lambda_{2}^{-}(\alpha) \ldots \ldots(4)
\end{aligned} \\
& \text { Since } \Omega_{f_{1}} \times \Omega_{f_{2}} \text { is ACB ideal of } \aleph \times \aleph, \text { then } \\
& \left(\widetilde{\mu}_{1}^{+} \times \widetilde{\mu}_{2}^{+}\right)\left(\beta_{1}, \beta_{2}\right) \geq \operatorname{rmin}\left\{\left(\widetilde{\mu}_{1}^{+} \times \widetilde{\mu}_{2}^{+}\right)\left(\left(\alpha_{1}, \alpha_{2}\right) *\left(\beta_{1}, \beta_{2}\right)\right),\left(\widetilde{\mu}_{1}^{+} \times \widetilde{\mu}_{2}^{+}\right)\left(\alpha_{1}, \alpha_{2}\right)\right\} \\
& =\operatorname{rmin}\left\{\left(\widetilde{\mu}_{1}^{+} \times \widetilde{\mu}_{2}^{+}\right)\left(\alpha_{1} * \beta_{1}, \alpha_{2} * \beta_{2}\right),\left(\widetilde{\mu}_{1}^{+} \times \widetilde{\mu}_{2}^{+}\right)\left(\alpha_{1}, \alpha_{2}\right)\right\}
\end{aligned}
$$

Put $\alpha_{1}=\beta_{1}=0$, then we have

$$
\begin{gathered}
\left(\widetilde{\mu}_{1}^{+} \times \widetilde{\mu}_{2}^{+}\right)\left(0, \beta_{2}\right) \geq \operatorname{rmin}\left\{\left(\widetilde{\mu}_{1}^{+} \times \widetilde{\mu}_{2}^{+}\right)\left(\left(0, \alpha_{2}\right) *\left(0, \beta_{2}\right)\right),\left(\widetilde{\mu}_{1}^{+} \times \widetilde{\mu}_{2}^{+}\right)\left(0, \alpha_{2}\right)\right\} \\
=\operatorname{rmin}\left\{\left(\widetilde{\mu}_{1}^{+} \times \widetilde{\mu}_{2}^{+}\right)\left(0, \alpha_{2} * \beta_{2}\right),\left(\widetilde{\mu}_{1}^{+} \times \widetilde{\mu}_{2}^{+}\right)\left(0, \alpha_{2}\right)\right\}
\end{gathered}
$$

and by equation (1), then

$\tilde{\mu}_{2}^{+}\left(\beta_{2}\right) \geq \operatorname{rmin}\left\{\tilde{\mu}_{2}^{+}\left(\alpha_{2} * \beta_{2}\right), \tilde{\mu}_{2}^{+}\left(\alpha_{2}\right)\right\}$. And

$$
\begin{gathered}
\left(\widetilde{\mu}_{1}^{-} \times \widetilde{\mu}_{2}^{-}\right)\left(\beta_{1}, \beta_{2}\right) \leq \operatorname{rmax}\left\{\left(\widetilde{\mu}_{1}^{-} \times \widetilde{\mu}_{2}^{-}\right)\left(\left(\alpha_{1}, \alpha_{2}\right) *\left(\beta_{1}, \beta_{2}\right)\right),\left(\widetilde{\mu}_{1}^{-} \times \widetilde{\mu}_{2}^{-}\right)\left(\alpha_{1}, \alpha_{2}\right)\right\} \\
=\operatorname{rmax}\left\{\left(\widetilde{\mu}_{1}^{-} \times \widetilde{\mu}_{2}^{-}\right)\left(\alpha_{1} * \beta_{1}, \alpha_{2} * \beta_{2}\right),\left(\widetilde{\mu}_{1}^{-} \times \widetilde{\mu}_{2}^{-}\right)\left(\alpha_{1}, \alpha_{2}\right)\right\}
\end{gathered}
$$

Put $\alpha_{1}=\beta_{1}=0$, then we have

$$
\begin{gathered}
\left(\widetilde{\mu}_{1}^{-} \times \widetilde{\mu}_{2}^{-}\right)\left(0, \beta_{2}\right) \leq \operatorname{rmax}\left\{\left(\widetilde{\mu}_{1}^{-} \times \widetilde{\mu}_{2}^{-}\right)\left(\left(0, \alpha_{2}\right) *\left(0, \beta_{2}\right)\right),\left(\widetilde{\mu}_{1}^{-} \times \widetilde{\mu}_{2}^{-}\right)\left(0, \alpha_{2}\right)\right\} \\
=\operatorname{rmax}\left\{\left(\widetilde{\mu}_{1}^{-} \times \widetilde{\mu}_{2}^{-}\right)\left(0, \alpha_{2} * \beta_{2}\right),\left(\widetilde{\mu}_{1}^{-} \times \widetilde{\mu}_{2}^{-}\right)\left(0, \alpha_{2}\right)\right\}
\end{gathered}
$$


and by using equation (2), we have $\tilde{\mu}_{2}^{-}\left(\beta_{2}\right) \leq \operatorname{rmax}\left\{\tilde{\mu}_{2}^{-}\left(\alpha_{2} * \beta_{2}\right), \tilde{\mu}_{2}^{-}\left(\alpha_{2}\right)\right\}$.

Also,

$$
\begin{gathered}
\left(\lambda_{1}^{+} \times \lambda_{2}^{+}\right)\left(\beta_{1}, \beta_{2}\right) \geq \min \left\{\left(\lambda_{1}^{+} \times \lambda_{2}^{+}\right)\left(\left(\alpha_{1}, \alpha_{2}\right) *\left(\beta_{1}, \beta_{2}\right)\right),\left(\lambda_{1}^{+} \times \lambda_{2}^{+}\right)\left(\alpha_{1}, \alpha_{2}\right)\right\} \\
=\min \left\{\left(\lambda_{1}^{+} \times \lambda_{2}^{+}\right)\left(\alpha_{1} * \beta_{1}, \alpha_{2} * \beta_{2}\right),\left(\lambda_{1}^{+} \times \lambda_{2}^{+}\right)\left(\alpha_{1}, \alpha_{2}\right)\right\}
\end{gathered}
$$

Put $\alpha_{1}=\beta_{1}=0$, then we have

$$
\begin{gathered}
\left(\lambda_{1}^{+} \times \lambda_{2}^{+}\right)\left(0, \beta_{2}\right) \geq \min \left\{\left(\lambda_{1}^{+} \times \lambda_{2}^{+}\right)\left(\left(0, \alpha_{2}\right) *\left(0, \beta_{2}\right)\right),\left(\lambda_{1}^{+} \times \lambda_{2}^{+}\right)\left(0, \alpha_{2}\right)\right\} \\
=\min \left\{\left(\lambda_{1}^{+} \times \lambda_{2}^{+}\right)\left(0, \alpha_{2} * \beta_{2}\right),\left(\lambda_{1}^{+} \times \lambda_{2}^{+}\right)\left(0, \alpha_{2}\right)\right\}
\end{gathered}
$$

and by using equation (3), we have

$\lambda_{2}^{+}\left(\beta_{2}\right) \geq \min \left\{\lambda_{2}^{+}\left(\alpha_{2} * \beta_{2}\right), \lambda_{2}^{+}\left(\alpha_{2}\right)\right\}$.

And

$$
\begin{gathered}
\left(\lambda_{1}^{-} \times \lambda_{2}^{-}\right)\left(\beta_{1}, \beta_{2}\right) \leq \max \left\{\left(\lambda_{1}^{-} \times \lambda_{2}^{-}\right)\left(\left(\alpha_{1}, \alpha_{2}\right) *\left(\beta_{1}, \beta_{2}\right)\right),\left(\lambda_{1}^{-} \times \lambda_{2}^{-}\right)\left(\alpha_{1}, \alpha_{2}\right)\right\} \\
=\max \left\{\left(\lambda_{1}^{-} \times \lambda_{2}^{-}\right)\left(\alpha_{1} * \beta_{1}, \alpha_{2} * \beta_{2}\right),\left(\lambda_{1}^{-} \times \lambda_{2}^{-}\right)\left(\alpha_{1}, \alpha_{2}\right)\right\}
\end{gathered}
$$

Put $\alpha_{1}=\beta_{1}=0$, then we have

$\left(\lambda_{1}^{-} \times \lambda_{2}^{-}\right)\left(0, \beta_{2}\right) \leq \max \left\{\left(\lambda_{1}^{-} \times \lambda_{2}^{-}\right)\left(\left(0, \alpha_{2}\right) *\left(0, \beta_{2}\right)\right),\left(\lambda_{1}^{-} \times \lambda_{2}^{-}\right)\left(0, \alpha_{2}\right)\right\}=\max \left\{\left(\lambda_{1}^{-} \times\right.\right.$ $\left.\left.\lambda_{2}^{-}\right)\left(0, \alpha_{2} * \beta_{2}\right),\left(\lambda_{1}^{-} \times \lambda_{2}^{-}\right)\left(0, \alpha_{2}\right)\right\}$ and by using equation (4), we have $\lambda_{2}^{-}\left(\beta_{2}\right) \leq \max \left\{\lambda_{2}^{-}\left(\alpha_{2} * \beta_{2}\right), \lambda_{2}^{-}\left(\alpha_{2}\right)\right\}$. And the condition $\left(\mathbf{B C}_{3}\right)$ is

$\left(\widetilde{\mu}_{1}^{+} \times \tilde{\mu}_{2}^{+}\right)\left(\left(\alpha_{1}, \alpha_{2}\right) \circ\left(\beta_{1}, \beta_{2}\right) \geq \operatorname{rmin}\left\{\left(\tilde{\mu}_{1}^{+} \times \widetilde{\mu}_{2}^{+}\right)\left(\alpha_{1}, \alpha_{2}\right),\left(\tilde{\mu}_{1}^{+} \times \tilde{\mu}_{2}^{+}\right)\left(\beta_{1}, \beta_{2}\right)\right\}\left(\tilde{\mu}_{1}^{+} \times\right.\right.$ $\left.\widetilde{\mu}_{2}^{+}\right)\left(\alpha_{1} \circ \beta_{1}, \alpha_{2} \circ \beta_{2}\right) \geq \operatorname{rmin}\left\{\left(\widetilde{\mu}_{1}^{+} \times \widetilde{\mu}_{2}^{+}\right)\left(\alpha_{1}, \alpha_{2}\right),\left(\widetilde{\mu}_{1}^{+} \times \widetilde{\mu}_{2}^{+}\right)\left(\beta_{1}, \beta_{2}\right)\right\}$

Put $\alpha_{1}=\beta_{1}=0$, then we have

$$
\left(\widetilde{\mu}_{1}^{+} \times \widetilde{\mu}_{2}^{+}\right)\left(0, \alpha_{2} \circ \beta_{2}\right) \geq \operatorname{rmin}\left\{\left(\widetilde{\mu}_{1}^{+} \times \widetilde{\mu}_{2}^{+}\right)\left(0, x_{2}\right),\left(\widetilde{\mu}_{1}^{+} \times \widetilde{\mu}_{2}^{+}\right)\left(0, \beta_{2}\right)\right\}
$$

and by using equation $(1)$, we have $\tilde{\mu}_{2}^{+}\left(\alpha_{2} \circ \beta_{2}\right) \geq \operatorname{rmin}\left\{\tilde{\mu}_{2}^{+}\left(\alpha_{2}\right), \tilde{\mu}_{2}^{+}\left(\beta_{2}\right)\right\}$

And

$$
\begin{gathered}
\left(\widetilde{\mu}_{1}^{-} \times \widetilde{\mu}_{2}^{-}\right)\left(\left(\alpha_{1}, \alpha_{2}\right) \circ\left(\beta_{1}, \beta_{2}\right) \leq \operatorname{rmax}\left\{\left(\widetilde{\mu}_{1}^{-} \times \widetilde{\mu}_{2}^{-}\right)\left(\alpha_{1}, \alpha_{2}\right),\left(\widetilde{\mu}_{1}^{-} \times \widetilde{\mu}_{2}^{-}\right)\left(\beta_{1}, \beta_{2}\right)\right\}\right. \\
\left(\widetilde{\mu}_{1}^{-} \times \widetilde{\mu}_{2}^{-}\right)\left(\alpha_{1} \circ \beta_{1}, \alpha_{2} \circ \beta_{2}\right) \leq \operatorname{rmax}\left\{\left(\widetilde{\mu}_{1}^{-} \times \widetilde{\mu}_{2}^{-}\right)\left(\alpha_{1}, \alpha_{2}\right),\left(\widetilde{\mu}_{1}^{-} \times \widetilde{\mu}_{2}^{-}\right)\left(\beta_{1}, \beta_{2}\right)\right\}
\end{gathered}
$$

Put $\alpha_{1}=\beta_{1}=0$, then we have

$$
\left(\widetilde{\mu}_{1}^{-} \times \widetilde{\mu}_{2}^{-}\right)\left(0, \alpha_{2} \circ \beta_{2}\right) \leq \operatorname{rmax}\left\{\left(\widetilde{\mu}_{1}^{-} \times \widetilde{\mu}_{2}^{-}\right)\left(0, \alpha_{2}\right),\left(\widetilde{\mu}_{1}^{-} \times \widetilde{\mu}_{2}^{-}\right)\left(0, \beta_{2}\right)\right\}
$$

And by using equation (2), we have $\tilde{\mu}_{2}^{-}\left(\alpha_{2} \circ \beta_{2}\right) \leq \operatorname{rmax}\left\{\tilde{\mu}_{2}^{-}\left(\alpha_{2}\right), \tilde{\mu}_{2}^{-}\left(\beta_{2}\right)\right\}$

Also, we have

$$
\begin{aligned}
& \left(\lambda_{1}^{+} \times \lambda_{2}^{+}\right)\left(\left(\alpha_{1}, \alpha_{2}\right) \circ\left(\beta_{1}, \beta_{2}\right) \geq \min \left\{\left(\lambda_{1}^{+} \times \lambda_{2}^{+}\right)\left(\alpha_{1}, \alpha_{2}\right),\left(\lambda_{1}^{+} \times \lambda_{2}^{+}\right)\left(\beta_{1}, \beta_{2}\right)\right\}\right. \\
& \left(\lambda_{1}^{+} \times \lambda_{2}^{+}\right)\left(\alpha_{1} \circ \beta_{1}, \alpha_{2} \circ \beta_{2}\right) \geq \min \left\{\left(\lambda_{1}^{+} \times \lambda_{2}^{+}\right)\left(\alpha_{1}, \alpha_{2}\right),\left(\lambda_{1}^{+} \times \lambda_{2}^{+}\right)\left(\beta_{1}, \beta_{2}\right)\right\}
\end{aligned}
$$

Put $\alpha_{1}=\beta_{1}=0$, then we have

$$
\left(\lambda_{1}^{+} \times \lambda_{2}^{+}\right)\left(0, \alpha_{2} \circ \beta_{2}\right) \geq \min \left\{\left(\lambda_{1}^{+} \times \lambda_{2}^{+}\right)\left(0, \alpha_{2}\right),\left(\lambda_{1}^{+} \times \lambda_{2}^{+}\right)\left(0, \beta_{2}\right)\right\}
$$

and by using equation (3), we have $\lambda_{2}^{+}\left(\alpha_{2} \circ \beta_{2}\right) \geq \min \left\{\lambda_{2}^{+}\left(\alpha_{2}\right), \lambda_{2}^{+}\left(\beta_{2}\right)\right\}$

And

$$
\begin{aligned}
& \left(\lambda_{1}^{-} \times \lambda_{2}^{-}\right)\left(\left(\alpha_{1}, \alpha_{2}\right) \circ\left(\beta_{1}, \beta_{2}\right) \leq \max \left\{\left(\lambda_{1}^{-} \times \lambda_{2}^{-}\right)\left(\alpha_{1}, \alpha_{2}\right),\left(\lambda_{1}^{-} \times \lambda_{2}^{-}\right)\left(\beta_{1}, \beta_{2}\right)\right\}\right. \\
& \left(\lambda_{1}^{-} \times \lambda_{2}^{-}\right)\left(\alpha_{1} \circ \beta_{1}, \alpha_{2} \circ \beta_{2}\right) \leq \max \left\{\left(\lambda_{1}^{-} \times \lambda_{2}^{-}\right)\left(\alpha_{1}, \alpha_{2}\right),\left(\lambda_{1}^{-} \times \lambda_{2}^{-}\right)\left(\beta_{1}, \beta_{2}\right)\right\}
\end{aligned}
$$

Put $\alpha_{1}=\beta_{1}=0$, then we have

$$
\left(\lambda_{1}^{-} \times \lambda_{2}^{-}\right)\left(0, \alpha_{2} \circ \beta_{2}\right) \leq \max \left\{\left(\lambda_{1}^{-} \times \lambda_{2}^{-}\right)\left(0, \alpha_{2}\right),\left(\lambda_{1}^{-} \times \lambda_{2}^{-}\right)\left(0, \beta_{2}\right)\right\}
$$

and by using equation (4), we have $\lambda_{2}^{-}\left(\alpha_{2} \circ \beta_{2}\right) \leq \max \left\{\lambda_{2}^{-}\left(\alpha_{2}\right), \lambda_{2}^{-}\left(\beta_{2}\right)\right\}$ 
Then, it follows that $\Omega_{\mathrm{f}_{2}}$ is ACB ideal of $\aleph$.

This completes the proof.

\section{References}

1. Prabpayak,C.; Leerawat. U. On ideals and congruence in KU-algebras. scientia Magna. 2009, 5, 1, 54-57.

2. Prabpayak, C.; Leerawat, U. On isomorphisms of KU-algebras. scientia magna (International Book Series). 2009. 5, 3, 25-31.

3. Mostafa, S.M.; Abd-Elnaby ,M.A. ; Yousef, M.M.M. Fuzzy ideals of KU-Algebras. Int. Math, Forum. 2011. 6, 63, 3139-3149.

4. Mostafa, S.M.; Abd-Elnaby ,M.A. ; Elgendy, O. R. Interval-valued Fuzzy KU-ideals in KU-algebras. Int. Math, Forum. 2011. 6, 64, 3151-3159.

5. Kareem, F.F.; Hasan,E.R. On KU-semigroups. International Journal of Science and Nature. 2018. 1, 9, 79-84.

6. Hasan, E. R.; Kareem, F.F. Fuzzy KU-Semi-Groups and Investigate Some Basic Properties, Journal of Engineering and Applied Science. 2018. 13, 18, 7739-7744.

7. Kareem, F.F.; Talib, S.A. Interval value fuzzy $k$-ideal of a KU-semigroup, Ibn AlHaitham Jour for Pure \& Appl. Sci. 2020. 33, 2, 95-106.

8. Jun, Y. B.; Kim, C. S.; Kang,M. S. Cubic subalgebras and ideals of BCK /BCI-algebras. Far East Journal of Mathematical Sciences, 2010. 2, 44, 239-250.

9. Jun, Y. B.; Kim C. S.; Yang, K.O. Cubic sets, Annals of Fuzzy Mathematics and Informatics. 2012. 1, 4, 83-98.

10. Yaqoob, N.; Mostafa, S. M.; Ansari, M. A. On cubic KU-ideals of KUalgebras. International Scholarly Research Notices, 2013.

11. Kareem F. F.; Awad,W. K. Cubic bipolar ideals of a semigroup in KU-algebra, Accepted for AIP Conference Ser. 2021. 